\title{
Prevalencia de depresión en cuidadores primarios de pacientes mayores de 60 años de edad y con alguna dependencia física
}

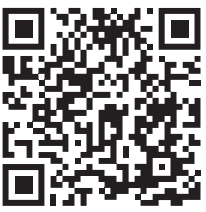

\author{
Prevalence of depression in primary caregivers of patients over \\ 60 years of age and with some physical dependency
}

Eli Nahim Becerra-Partida,* Carlos Andrés Villegas Pachecoł

\begin{abstract}
RESUMEN
Objetivo: Conocer la prevalencia de depresión que existe en los cuidadores primarios de pacientes con dependencia física y mayores de 60 años. Material y métodos: Estudio transversal, no comparativo, descriptivo y observacional. Muestreo según la fórmula de probabilidad para poblaciones definidas. Este estudio incluyó a todos los cuidadores primarios de pacientes mayores de 60 años con dependencia física que acudieron a la Unidad de Medicina Familiar (UMF) No. 78 del Instituto Mexicano del Seguro Social (IMSS) durante un periodo de tres meses consecutivos. Se utilizó la escala de autoevaluación para la depresión de Zung y la encuesta de Barthel. Las variables cualitativas se describieron mediante frecuencia simple y porcentajes. La descripción de las variables cuantitativas se realizó de acuerdo con la media, mediana, moda y desviación estándar. El análisis estadístico se realizó con el programa SPSS versión 22 acorde con el diseño del estudio. Resultados: La muestra final fue de 238 participantes; se observó una prevalencia de depresión en 127 del total de los encuestados; de éstos el 50\% cursa con depresión leve, $8 \%$ con depresión moderada y no se encontró algún paciente con depresión severa. Conclusiones: La prevalencia de depresión en los cuidadores primarios de pacientes con algún grado de dependencia física es significativa entre lo percibido por los cuidadores primarios y lo evaluado por el médico. El género más afectado fue el femenino y se encontró el predominio de dependencia física moderada.
\end{abstract}

Palabras clave: Depresión, escala de autoevaluación de Zung, escala de Barthel, cuidadores primarios.

\begin{abstract}
Objective: To know the prevalence of depression in primary caregivers of patients over 60 years of age with physical dependence. Material and methods: Cross-sectional, non-comparative, descriptive and observational study. Sampling according to the probability formula for defined populations. It included all primary caregivers of patients over 60 years of age with physical dependence who attended the Family Medicine Unit (UMF) No. 78 of the Instituto Mexicano del Seguro Social (IMSS) for a period of three consecutive months. The self-assessment scale for Zung depression and the Barthel survey were used. Qualitative variables were described by simple frequency and percentages. The description of the quantitative variables was made according to the mean, median, mode and standard deviation. The statistical analysis was performed with the SPSS version 22 program according to the study design. Results: The final sample was 238 participants where a prevalence of depression was observed in 127 of the 238 subjects, of which $50 \%$ presented mild depression, $8 \%$ moderate depression and no patient with severe depression was found. Conclusions: The prevalence of depression in primary caregivers of patients with some degree of physical dependence is significant between what is perceived by primary caregivers and what is evaluated by the doctor. The most affected gender was female and a moderate physical dependence prevalence was found.
\end{abstract}

Keywords: Depression, Zung self-assessment scale, Barthel scale, primary caregivers.
* Médico Familiar adscrito al Servicio de Atención Médica Continua. Asesor del Curso de Especialización en Medicina Familiar e Investigador. Doctorante en Bioética y Derechos Humanos del Instituto de Ética y Terapia de Jalisco. * Médico Residente de Medicina Familiar.

Unidad de Medicina Familiar No. 78 del Instituto Mexicano del Seguro Social, Guadalajara, Jalisco, México.

Correspondencia: ENBP, elinahim@hotmail.es Conflicto de intereses: Los autores declaran que no tienen.

Citar como: BecerraPartida EN, Villegas PCA. Prevalencia de depresión en cuidadores primarios de pacientes mayores de 60 años de edad y con alguna dependencia física. Rev CONAMED. 2020; 25(4): 161-166. https:// dx.doi.org/10.35366/97335 Financiamiento: Este trabajo no contó con financiamiento. 


\section{INTRODUCCIÓN}

Las estimaciones del Consejo Nacional de Población (CONAPO) consideran que en 2017 residieron en el país 12,973,411 personas de 60 años o más. La esperanza de vida de la población ha aumentado considerablemente (75.3 años en 2017) y la fecundidad es cada vez menor (2.21 hijos por mujer), por lo que el peso relativo de los adultos mayores toma una mayor relevancia en la estructura por edad. Entre 1970 y 1990 el porcentaje de adultos mayores respecto de la población total pasó de 5.6 a 6.2\%; para 2017 dicho porcentaje fue de $10.5 \%$.

En el 2012, la Encuesta Nacional de Salud y Nutrición reportó que una cuarta parte de los adultos mayores (26.9\%) se encuentra con algún tipo de discapacidad; esto se traduce a una reducción en las actividades básicas de la vida diaria (bañarse, caminar, acostarse o levantarse de la cama y vestirse) y las actividades instrumentales de la vida diaria (compra de alimentos, administración de medicamentos, manejo de dinero y preparación de alimentos). En ambas situaciones se encuentra una tendencia ascendente conforme aumenta la edad. ${ }^{2}$

Se considera como persona dependiente al individuo que, por razones de edad, enfermedad o discapacidad, ha perdido la autonomía física, sensorial, mental o intelectual y que requiere permanente atención de otra persona para sus actividades diarias. Una gran parte de los cuidados básicos e instrumentales para estas personas se pone a cargo de los denominados cuidadores informales, quienes no reciben retribución económica por dicha labor y entre los que destacan los cuidados prestados por su familia. ${ }^{3-5}$ La familia dispone de recursos como pueden ser la cohesión, adaptabilidad, normas, valores y conductas para afrontar las crisis familiares tanto normativas como paranormativas; sin embargo, la presencia de una enfermedad incapacitante en uno de los miembros exige la disposición del cuidado a otro de los miembros de la familia, y se inicia así una alteración en la dinámica familiar que, si no se supera de modo satisfactorio, se puede desarrollar una serie de alteraciones entre las que se incluye la depresión. Esto repercute en la salud física del cuidador, su estado de ánimo y en la modificación de la percepción del sufrimiento y del dolor del enfermo a su cuidado. ${ }^{6}$

La Organización Mundial de la Salud, en al año de 1999, definió al cuidador primario como «...la persona del entorno de un paciente que asume voluntariamente el papel de responsable del mismo en un amplio sentido: este individuo está dispuesto a tomar decisiones por el paciente, decisiones para el paciente, y a cubrir las necesidades básicas del mismo, ya sea de manera directa o indirecta...». ${ }^{7}$

Estas personas deben contar con características específicas como: responsabilidad, paciencia, disciplina, adaptabilidad a los cambios, apoyo emocional al paciente y tiempo para brindar acompañamiento. Además, se puede observar una mayor disposición para asumir el tratamiento de una enfermedad crónica en aquellos pacientes que cuentan con el apoyo de un familiar. ${ }^{8}$

Se estima que $88 \%$ del tiempo dedicado a cuidar a los pacientes dependientes recae en el cuidador principal. Las mujeres no sólo asumen de forma mayoritaria el rol de cuidadores principales, sino que también se encargan de las tareas más pesadas y demandantes, además de que dedican más tiempo a cuidar que los hombres, lo que genera una clara inequidad de género. ${ }^{9}$

Conforme aumenta la carga de trabajo de la persona junto con la responsabilidad de los cuidados primarios, también se incrementa el riesgo de deterioro de su salud y su calidad de vida. ${ }^{10}$ La responsabilidad de cuidar a una persona con una enfermedad crónica implica que el cuidador maneje eventos vitales, los cuales repercuten en la calidad de vida de la persona dependiente e implica mucho tiempo para ella misma; esto se realiza de manera incondicional en la mayoría de los casos, dejando de lado sus propias necesidades y requerimientos personales, lo que afecta su calidad de vida.,11

El resultado del estrés crónico, que es producido por la lucha diaria contra la monotonía, la enfermedad y la sensación de falta de control, entre otras cosas, se caracteriza por actitudes y pensamientos negativos (en algunos casos graves) hacia el enfermo, tales como los siguientes que encontramos en nuestro estudio: desmotivación, depresión-angustia, trastornos psicosomáticos, fatiga y agotamiento no ligado al esfuerzo; también 
irritabilidad, despersonalización y deshumanización, comportamientos estereotipados con ineficiencia en resolver los problemas reales y agobio continuo con sentimientos de ser desbordados por la situación. 12 Esta situación genera muchas reacciones afectivas y emocionales, tales como tensión, ira, tristeza y culpabilidad. Todo comienza con un desgaste emocional, físico y económico hasta llegar el momento en el que el cuidador presenta gran incidencia de trastornos psicológicos como estrés, ansiedad, depresión, etcétera. ${ }^{13}$

Diversas investigaciones han demostrado que brindar cuidado puede afectar negativamente la salud psicológica del cuidador. Se ha reportado en la literatura que ciertos factores como la edad avanzada, la condición laboral desfavorable y el inadecuado apoyo social aumentan el riesgo de presentar problemas. ${ }^{14}$

Adicionalmente ha habido un aumento en la aparición del trastorno depresivo entre los cuidadores que se hacen responsables del cuidado del enfermo.

Considerando todo lo anterior, en la Unidad de Medicina Familiar número 78 se desconoce la prevalencia de depresión en los cuidadores primarios de los pacientes con alguna dependencia física y mayores de 60 años; ya que no existe un antecedente previo, consideramos que realizar este estudio es un oportuno comienzo que nos aportará gran valor estadístico y clínico a nuestra población para implementar medidas de prevención y planear estrategias que mejoren la calidad de vida.

\section{MATERIAL Y MÉTODOS}

Diseño: se trató de un estudio trasversal, descriptivo, no probabilístico, observacional y prospectivo, el cual fue realizado en la UMF número 78 del IMSS durante un periodo de tres meses posteriores a la autorización del Comité Local de Investigación y Ética en Salud (CLIES).

Participantes: cuidadores primarios de pacientes con alguna dependencia física y mayores de 60 años, los cuales acudieron a la Unidad de Medicina Familiar Número 78; se consideró una muestra de éstos, con un nivel de confianza de 95\%, p = 0.5 como máxima varianza permisible. El resultado fue de 238 pacientes.
Los criterios de inclusión fueron: que fueran derechohabientes del IMSS mayores de edad y que actualmente sean cuidadores de un paciente mayor de 59 años de edad, además de que acepten participar en el estudio y que firmen la carta de consentimiento informado. Los criterios de exclusión fueron: cuidadores que atiendan a más de dos personas y que no deseen participar en el estudio. Los criterios de eliminación fueron: no contestar el cuestionario completo y no firmar la carta de consentimiento informado.

Instrumento: para valorar la depresión que presentó el paciente se aplicó la escala de autoevaluación para la depresión de Zung; ésta cuenta con 20 ítems evaluados en una escala de 1-4, con lo que se obtiene un rango de calificación de 20-80.

La dependencia del paciente del cual se hace cargo el cuidador se midió por medio de la escala de Barthel; ésta mide la capacidad de una persona en 10 actividades realizadas en la vida diaria; tiene un puntaje de 0-10 con el que se puede obtener un máximo de 100 puntos.

Análisis estadístico: se hizo un vaciado de información en una hoja del programa Excel 2010 previamente elaborada para este estudio. Después, los resultados se analizaron con el software estadístico SPSS versión 22.0. Las variables cualitativas se describieron mediante frecuencia simple y porcentajes. La descripción de las variables cuantitativas se realizó de acuerdo con la media, mediana, moda y desviación estándar.

Aspectos éticos: el protocolo de investigación fue autorizado por el Comité Local de Investigación en Salud 1306 de la delegación Jalisco del IMSS, con número de registro R-2019-1306-093.

\section{RESULTADOS}

Se encuestó un total de 238 pacientes, los cuales afirmaron ser cuidadores primarios de personas mayores de 60 años con algún tipo de dependencia física y que cumplieron los criterios de inclusión. Se encontraron los datos sociodemográficos presentados en la Tabla 7.

Al aplicar la escala de autoevaluación de Zung se encontró una prevalencia de depresión del 53.4\% (Tabla 2). La aplicación de la escala de Barthel mostró, respecto del grado de dependencia física 
del paciente que está a su cuidado, lo siguiente: cinco pacientes con dependencia física leve, lo que representa 2.1\%; 118 pacientes con dependencia moderada, que representa 49.6\%; 86 pacientes con dependencia severa, que representa $36.1 \%$ y 17 pacientes con dependencia total, que representa 7.1\%; 12 pacientes son independientes, lo que representa $5 \%$.

Lo anterior muestra un riesgo para el cuidador primario, que implicará un incremento de las complicaciones en pacientes mayores de 60 años, tales como son: úlceras por presión, malnutrición, aumento de riesgo de complicaciones en síndromes geriátricos e incremento de riesgo de caídas y sus consecuencias (eventos vasculares cerebrales, fracturas e infecciones de vías respiratorias y urinarias).

Tabla 1: Características sociodemográficas.

\begin{tabular}{lcc}
\hline Variable & $\mathrm{n}$ & $\%$ \\
\hline Género & & \\
Femenino & 152 & 63.9 \\
$\quad$ Masculino & 86 & 36.1 \\
Estado civil & & \\
Soltero & 43 & 18.1 \\
Casado & 136 & 57.1 \\
Unión libre & 8 & 3.4 \\
Divorciado & 34 & 14.3 \\
Viudo & 17 & 7.1 \\
Escolaridad & & \\
Primaria & 48 & 20.2 \\
Secundaria & 59 & 24.8 \\
Preparatoria & 79 & 33.2 \\
Universidad & 38 & 16.0 \\
Sin estudios & 14 & 5.9 \\
Edad (años) & & \\
18-30 & 21 & 8.8 \\
31-40 & 49 & 20.6 \\
$41-50$ & 78 & 32.8 \\
51-60 & 36 & 15.1 \\
61-70 & 36 & 15.1 \\
71-80 & 12 & 5.0 \\
81-85 & 6 & 2.5 \\
\hline
\end{tabular}

Tabla 2: Prevalencia de depresión.

\begin{tabular}{lrr}
\hline Variable & $\mathrm{n}$ & $\%$ \\
\hline Depresión leve & 119 & 50.0 \\
Depresión moderada & 8 & 3.4 \\
No depresión & 111 & 46.6 \\
Total & 238 & 100.0 \\
\hline
\end{tabular}

De esta manera, es trascendente la intervención primaria por el médico familiar, así como la aplicación de las escalas a los cuidadores y la identificación de los criterios diagnósticos de depresión en el cuidador primario, para que, de esta manera, se inicie el tratamiento oportuno y transdisciplinario individualizado a cada paciente. Así se evitará el aumento en la tasa de mortalidad por las complicaciones antes mencionadas en el adulto mayor y las consecuencias en potencia que puedan generar la depresión en el cuidador primario. ${ }^{15}$

\section{DISCUSIÓN}

Si se considera el aumento de la expectativa de vida, las enfermedades crónico-degenerativas han aumentado la frecuencia de dependencia física de los pacientes, por lo que se desprende un incremento en la participación de los cuidadores primarios, requiriendo, en algunos casos, tiempo completo. 11,16

Se puede observar que, a pesar de que la dependencia física por alguna enfermedad crónica se encuentre en línea ascendente, no existe consejería estipulada para el cuidador primario, ya que vive la enfermedad con el paciente directamente, formando una inestabilidad entre las partes estrechamente ligadas a la vida del cuidador. ${ }^{9}$ El cuidador primario no comprende esta circunstancia compleja en la que se crea una mala relación con su familia y con el paciente, lo que puede llevar, en el peor de los casos, a convertirlo en un paciente dependiente por la poca atención que se le da a su propia salud. ${ }^{10}$

Este estudio es equiparable con otros estudios como los de Jafet Méndez, en los que la edad promedio del cuidador primario es de 43 años, 
coincidiendo con nosotros en una edad promedio de 49 años para este estudio. ${ }^{17}$ Además, coincide con otros estudios en la prevalencia mayor, que va de depresión leve a moderada, tal como en el estudio de Navarro Sandoval. ${ }^{5}$

\section{CONCLUSIONES}

Durante el proceso del estudio se apreció el interés y compromiso de los cuidadores primarios de pacientes que tienen algún grado de dependencia física respecto a la importancia de su participación en la salud del enfermo, así como en su propia salud.

Podemos concluir que la depresión leve fue la más frecuente, siendo estadísticamente significativa. La depresión moderada seguida de la depresión severa, recibieron un menor porcentaje, pero aun así representan un factor de riesgo significativo en la atención del paciente al cuidado, así como la disminución en la salud del cuidador primario. El promedio de edad fue de 49 años, con predominio del sexo femenino; el estado civil principalmente fue de casado y la escolaridad predominante fue de preparatoria.

Sugerimos realizar otros estudios de investigación con otros diseños que puedan determinar la relación causal, en el que el tamaño de la muestra sea mayor y el tipo de muestreo sea aleatorio para aumentar la validez.

\section{AGRADECIMIENTOS}

Al Instituto Mexicano del Seguro Social por ser el pilar de nuestro sistema de salud.

\section{BiblogRAFÍA}

1. Instituto Nacional de Estadística y Geografía [Internet]. Aguascalientes (ACS): Estadísticas a propósito del Día Mundial de la Población; 2017. [Consultado noviembre 2018]. Disponible en: https://www.inegi.org.mx/contenidos/ saladeprensa/aproposito/2017/poblacion2017_Nal.pdf

2. Gutiérrez JP, Rivera-Dommarco J, Shamah-Levy T, Villalpando-Hernández S, Franco A, Cuevas-Nasu L et al. Encuesta Nacional de Salud y Nutrición 2012. Resultados Nacionales. Cuernavaca, México: Instituto Nacional de Salud Pública (MX); 2012.

3. López MJ, Orueta R, Gómez-Caro S, Sánchez A, Carmona J, Alonso FJ. El rol de cuidador de personas dependientes y sus repercusiones sobre su calidad de vida y su salud. Rev Clin Med Fam. 2009; 2 (7): 332-339.

4. López-Casanova P, Rodríguez-Palma M, HerreroDíaz MA. Perfil social de los cuidadores familiares de pacientes dependientes ingresados en el Hospital General Universitario de Elche. Gerokomos. 2009; 20 (4): 167-171.

5. Orueta-Sánchez R, Gómez-Calcerrada RM, Gómez-Caro S, Sánchez-Oropesa A, López-Gil MJ, Toledano-Sierra P. Impacto sobre el cuidador principal de una intervención realizada a personas mayores dependientes. Aten Primaria. 2017; 43 (9): 490-496.

6. Dueñas E, Martínez MA, Morales B, Muñoz C, Viáfara AS, Herrera JA. Síndrome del cuidador de adultos mayores discapacitados y sus implicaciones psicosociales. Colomb Med. 2006; 37 (2) S1: 31-38.

7. WHO Ageing and Health Programme \& Milbank Memorial Fund. Hacia un consenso internacional sobre la política de atención de salud a largo plazo en el envejecimiento. Ginebra: Organización Mundial de la Salud; 2000. Disponible en: https://apps.who.int/iris/ handle/10665/66542

8. Archury DM, Castaño HM, Gómez LA, Guevara NM. Calidad de vida de los cuidadores de pacientes con enfermedades crónicas con parcial dependencia. Investigación en Enfermería: Imagen y Desarrollo [Internet]. 2011; 13 (1): 27-46. Recuperado de: https://www.redalyc.org/articulo. oa?id=145221282007

9. Rivera MH, Dávila MR, González PAA. Calidad de vida de los cuidadores primarios de pacientes geriátricos de la Clínica de Medicina Familiar Dr. Ignacio Chávez, ISSSTE. Rev Esp Med Quir. 2011; 16 (1): 27-32.

10. Larañaga I, Martín U, Bacigalupe A, Begiristáin JM, Valderrama MJ, Arregi B. Impacto del cuidado informal en la salud y la calidad de vida de las personas cuidadoras: análisis de las desigualdades de género. Gac Sanit. 2008; 22 (5): 443-450.

11. Alfaro-Ramírez del Castillo Ol, Morales-Vigil T, VázquezPineda F, Sánchez-Román S, Ramos-del Río B, GuevaraLópez U. Sobrecarga, ansiedad y depresión en cuidadores primarios de pacientes de dolor crónico y terminales. Rev Med Inst Mex Seguro Soc. 2008; 46 (5): 485-494.

12. Palacios-Espinosa $X$, Jiménez-Solanilla KO. Estrés y depresión en cuidadores informales de pacientes con trastorno afectivo bipolar. Avances en Psicología Latinoamericana. 2008; 26 (2): 195-210.

13. Peinado Al, Garcés de los Fayos EJ. Burnout en cuidadores principales de pacientes con Alzheimer: el síndrome del asistente desasistido. Anales de Psicología [Internet]. 1998; 14 (1): 83-93. Recuperado de: https://www.redalyc.org/ articulo.oa?id=16714108

14. Rodríguez-Agudelo Y, Mondragón-Maya A, Paz-Rodríguez F, Chávez-Oliveros M, Solís-Vivanco R. Variables asociadas con ansiedad y depresión en cuidadores de pacientes con 
enfermedades neurodegenerativas. Arch Neurocien. 2010; 15 (1): 25-30.

15. American Psychiatric Association. Manual diagnóstico y estadístico de trastornos mentales. 5a edición. Arlington, VA: American Psychiatric Association Publishing; 2016.

16. Rivas-Acuña $\vee$, García-Barjau H, Cruz-León A, MoralesRamón F, Enríquez-Martínez RM, Román-Álvarez J.
Prevalencia de ansiedad y depresión en las personas con diabetes mellitus tipo 2. Salud en Tabasco [Internet]. 2017; 17 (1,2): 30-35. Recuperado de: https://www.redalyc.org/ articulo.oa?id=48721182005

17. Domínguez JA, Ruíz M, Gómez I, Gallego E, Valero J, Izquierdo MT. Ansiedad y depresión en cuidadores de pacientes dependientes. Semergen. 2012; 38 (1): 16-23. 